\title{
Effects of Dietary Intake of Isoflavone Aglycone-rich Fermented Soybeans on Bone Metabolism in Ovariectomized Rats
}

\author{
Yuki Okabe $^{* a}$ and Hiroyuki Tanimoto ${ }^{b}$ \\ ${ }^{a}$ Research Institute for Health Fundamentals, Ajinomoto Co., Inc., 1-1 Suzuki-cho, Kawasaki, 210-8681, Japan and ${ }^{b}$ Seasonings \\ Dept., Food Products Company, Ajinomoto Co., Inc., 1-15-1 Kyobashi, Chuo-ku, Tokyo, 104-8315, Japan
}

(Received November 21, 2007; Accepted February 27, 2008; Published online March 3, 2008)

\begin{abstract}
We compared the effect of dietary intake of isoflavone aglycone-rich fermented soybeans (FS) with that of glucoside-rich non-fermented soybeans (NFS) on bone metabolism, using ovariectomized (OVX) rats. Female Sprague-Dawley rats were given OVX or a sham operation at 8 weeks of age. After a 2-week recovery, shamoperated rats were given a NFS diet for 57 days, while OVX rats were given the NFS or FS diet (Sham-NFS, OVX-NFS, and OVX-FS group, $n=10)$. The two diets had the same level of total isoflavone $(228 \mu \mathrm{mol} / 100 \mathrm{~g})$. The percentage of isoflavone aglycone to total isoflavone was $100 \%$ in the FS diet, and approximately $5 \%$ in the NFS diet. Tibial bone calcium and phosphorus contents were significantly lower in the OVX-NFS group than in the Sham-NFS group. This reduction was significantly prevented in the OVX-FS group. Tibial trabecular bone density was also significantly reduced by OVX, but the reduction tended to be prevented in the OVX-FS group $(p=0.1)$. The level of urinary deoxypyridinoline (DPD), a bone resorption marker, was significantly increased by OVX; this increase was significantly prevented in the OVX-FS group. Moreover, urinary total isoflavone levels tended to be higher in the OVX-FS group than in the OVX-NFS group $(p=0.1)$. In conclusion, this study suggested that intake of FS prevented excessive bone resorption and bone loss following OVX in rats. It was also suggested that these beneficial effects on bone resulted from higher absorption of isoflavone aglycone.
\end{abstract}

Key words — isoflavone aglycone, fermented soybean, bone metabolism, ovariectomized, rat

\section{INTRODUCTION}

Osteoporosis has become a major health problem worldwide. Especially in women, estrogen deficiency after menopause is a major risk for osteoporosis. ${ }^{1)}$ Hormone replacement therapy has been used for many years and can be quite effective. However, there is currently a controversy over using such estrogen preparations because of their possible side effects. ${ }^{2,3)}$ Thus, it is necessary to develop alternatives, such as natural food products, which are more moderate and have no side effects.

Isoflavones are flavonoids that are contained mainly in soybeans. They are structurally similar to estrogen, and have weak binding abilities to estrogen receptors. ${ }^{4)}$ Thus, they exhibit moderate estrogen-like effects. Many studies have been

\footnotetext{
${ }^{*}$ To whom correspondence should be addressed: Research Institute for Health Fundamentals, Ajinomoto Co., Inc., 1-1 Suzuki-cho, Kawasaki, 210-8681, Japan. Tel.: +81-44-2105767; Fax: +81-44-211-7609; E-mail: yuki_okabe@ajinomoto. com
}

reported the beneficial effects of isoflavones on bone in animals ${ }^{5,6)}$ and humans. ${ }^{7,8)}$ Isoflavones are present in soybeans and most soy foods primarily in the glucose-conjugated form (glucoside). ${ }^{9)}$ Isoflavone is not absorbed in this form. Once ingested, glucoside must be hydrolyzed to absorbable aglycone by $\beta$-glucosidase in the intestinal microflora. ${ }^{10,11)}$ Therefore, it is commonly considered that aglycone is absorbed faster than glucoside. ${ }^{12)}$ On the other hand, the bioavailability of aglycone and glucoside remains controversial. Izumi et al. ${ }^{13)}$ reported that the amount of isoflavones absorbed is higher for the aglycone than the glucoside form. Kano et al. ${ }^{14)}$ reported that the amount of isoflavones absorbed was higher in aglyconerich fermented soymilk than in glucoside-rich nonfermented soymilk; however, other studies have reported no significant difference in the bioavailability of aglycone and glucoside forms. $\left.{ }^{15}, 16\right)$ These apparently different results may be partly because of the amount of isoflavone intake or the food matrix containing the isoflavone. ${ }^{12)}$ For example, Setchell 
et $a l .{ }^{17)}$ suggested that intake of large doses of isoflavones at one time did not necessarily enhance the bioavailability of isoflavones from the pharmacokinetic view. Cassidy et al. ${ }^{18)}$ reported the greater bioavailability of isoflavones in soymilk than in textured vegetable protein. There has not been enough systematic research considering the various factors potentially affecting the pharmacokinetics of isoflavones.

Nonetheless, it makes sense to speculate that aglycone is superior to glucoside in that its first step of absorption is independent of intestinal microflora, which can be influenced by many factors, such as aging, food, mental condition, and so on. ${ }^{19,20)}$

We developed a new type of fermented soybeans produced by fermenting soybeans with koji (Aspergillus oryzae) under salt-free conditions. The product is unique in that more than $90 \%$ of isoflavones are present in the aglycone form. This is because the activity of koji considerably increases under salt-free conditions, which enables hydrolyzation of isoflavone to aglycone in a very short time. ${ }^{21)}$ At present, we call this new material "isoflavone aglycone-rich fermented soybeans" (FS). Traditional fermented soy foods, like miso, natto, and tempeh, have isoflavone aglycone, too, but at lower rates. Miso has about $65 \%$ aglyconeform isoflavone, ${ }^{22)}$ natto has about $9 \%,{ }^{22)}$ and tempeh has about $37 \%{ }^{23)}$ Therefore, it is speculated that FS will exhibit superior beneficial effects on bone through the high absorption of isoflavone aglycone. To our knowledge, there have been few studies clearly showing the superior bone-protective effects of aglycone or aglycone-rich soy foods as compared to glucoside or glucoside-rich soy foods.

In this research, we compared the effects of isoflavone aglycone-rich FS with isoflavone glucoside-rich non-fermented soybeans (NFS) on bone metabolism in ovariectomized (OVX) rats. We also investigated the isoflavone absorption by prolonged intake of FS and NFS.

\section{MATERIALS AND METHODS}

Animals — Female Sprague-Dawley rats (7 weeks old) were obtained from Charles River Laboratories Japan (Yokohama, Japan). All rats were kept in a room with controlled lighting (lights on 08:00-20:00), temperature $\left(22 \pm 3^{\circ} \mathrm{C}\right)$, and humidity $(50 \pm 20 \%)$. They were individ- ually housed in standard stainless steel cages $(225 \times 185 \times 200 \mathrm{~mm})$. Rats were given free access to water and commercial diets (CRF1; Oriental Yeast, Tokyo, Japan). After a 7-day adaptation period, rats were given a bilateral OVX or sham operation. In the sham-operated rats, both ovaries were handled, but not removed. After a 14-day recovery period, experimental diets were initiated. The rats were maintained in accordance with the guidelines of the ethical committee for the Animal Experiments of Ajinomoto Co. Inc. (Kawasaki, Japan) Institute.

Diets — FS was produced in a similar way as miso, but without using salt. Soybeans were steamed and fermented with koji. The obtained paste was vacuum drum dried, then milled into powder. All of the isoflavones in the FS powder were aglycones (Table 1). At the same time, NFS was produced by steaming the same soybeans, and then drying in the same way. As a result, 5\% of its isoflavones were aglycones, and $95 \%$ were glucosides (Table 1). Isoflavones were analyzed by HPLC-UV, using the method of Kudou et al. ${ }^{24)}$

FS powder $(39.5 \%)$ or NFS powder $(40.7 \%)$ was added to the diets so that both diets had the same amount of isoflavones $(228 \mu \mathrm{mol} / 100 \mathrm{~g}$ diet $)$. Both diets were adjusted to contain $0.5 \%$ calcium (Ca), $0.3 \%$ phosphorus $(\mathrm{P}), 10.7 \%$ fat, and $15.5 \%$ protein. The composition of the diets is shown in Table 2.

Experimental Protocol — After a 14-day postoperative recovery, sham-operated rats were given

Table 1. Isoflavone Concentration in NFS and FS powder

\begin{tabular}{lcc}
\hline \hline$\mu$ mol/100g & $\begin{array}{c}\text { NFS } \\
\text { powder }\end{array}$ & $\begin{array}{c}\text { FS } \\
\text { powder }\end{array}$ \\
\hline daidzin & 173.1 & $-^{a)}$ \\
genistin & 295.1 & - \\
glycitin & 6.4 & - \\
acetyldaidzin & 17.4 & - \\
acetylgenistin & 34.8 & - \\
acetylglycitin & - & - \\
malonyldaidzin & - & - \\
malonylgenistin & 6.1 & - \\
malonylglycitin & - & - \\
daidzein & 11.2 & 230.3 \\
genistein & 15.6 & 338.9 \\
glycitein & - & 5.8 \\
\hline total isoflavone & 559.6 & 575.0 \\
total glucoside & 532.9 & - \\
total aglycone & 26.8 & 575.0 \\
\hline
\end{tabular}

a) Detection limit was $0.5 \mathrm{mg} / 100 \mathrm{~g}$. 
Table 2. Composition of NFS and FS Diet

\begin{tabular}{|c|c|c|}
\hline \multirow[t]{2}{*}{ Ingredients $(\mathrm{g} / 100 \mathrm{~g})$} & \multicolumn{2}{|c|}{ Diet } \\
\hline & NFS & $\mathrm{FS}$ \\
\hline dextrin & 38.2 & 39.5 \\
\hline sucrose & 10.0 & 10.0 \\
\hline soy oil ${ }^{a)}$ & 0 & 0.2 \\
\hline AIN93G mineral mixture ${ }^{b \text { ) }}$ & 3.5 & 3.5 \\
\hline AIN93 vitamin mixture ${ }^{c)}$ & 1.0 & 1.0 \\
\hline BHT & 0.0014 & 0.0014 \\
\hline cellulose & 5.0 & 5.0 \\
\hline $\mathrm{CaCo}^{a)}$ & 1.049 & 1.057 \\
\hline $\mathrm{KH} 2 \mathrm{PO} 4^{a}$ & 0.158 & 0.178 \\
\hline NFS powder & 40.7 & 0 \\
\hline FS powder & 0 & 39.5 \\
\hline defatted soy protein ${ }^{a, d)}$ & 0.487 & 0 \\
\hline Total & 100 & 100 \\
\hline \multicolumn{3}{|l|}{ Calculated Values } \\
\hline Fat $(\%)$ & 10.7 & 10.7 \\
\hline Protein $(\%)$ & 15.5 & 15.5 \\
\hline Carbohydrate (\%) & 65.0 & 64.4 \\
\hline Isoflavones $(\mu \mathrm{mol} / 100 \mathrm{~g})$ & 228 & 228 \\
\hline aglycone & 11 & 228 \\
\hline glycoside & 217 & 0 \\
\hline $\mathrm{Ca}(\%)$ & 0.5 & 0.5 \\
\hline $\mathrm{P}(\%)$ & 0.3 & 0.3 \\
\hline
\end{tabular}

a) Contents were adjusted and added so that the total amounts of fat, protein, $\mathrm{Ca}$, and $\mathrm{P}$ in the two diets were equal (FS and NFS themselves were unequal). $b$ ) Sucrose was substituted for $\mathrm{CaCo}_{3}$ and $\mathrm{KH}_{2} \mathrm{PO}_{4}$. c) Contained $25 \%$ choline bitartrate. d) Isoflavone-free defatted soy protein $(<1 \mu \mathrm{mol} / 100 \mathrm{~g})$.

the NFS diet (Sham-NFS) for 57 days. Half of the OVX rats were given the NFS diet (OVX-NFS), and the other half was given the FS diet (OVS-FS). Each group had 10 rats. The Sham-NFS group was given free access to the diet. The OVX-NFS and OVX-FS groups were pair-fed.

After 3 weeks of the experimental diet, blood and urine samples were collected to analyze the markers of bone turnover. On day 19, blood samples were collected from the subclavian vein. Serum samples were obtained by centrifugation at $1500 \times g$ at $4^{\circ} \mathrm{C}$ for $15 \mathrm{~min}$ and stored at $-70^{\circ} \mathrm{C}$ until analysis for osteocalcin. On day 24, urine samples were collected for $24 \mathrm{hr}$, using metabolic cages, and stored at $-20^{\circ} \mathrm{C}$ until analysis for deoxypyridinoline (DPD).

On day 53, urine samples were collected for $24 \mathrm{hr}$ and stored at $-20^{\circ} \mathrm{C}$ until analysis for isoflavones. On the final day of the experiment, after overnight fasting, rats were anaesthetized with diethyl ether. Blood samples were collected from the inferior vena cava, and then the rats were sacrificed. The organs were removed immediately. The right tibial bone was carefully cleaned of surrounding tissue, and kept in $70 \%$ ethanol until the bone density analysis. The left tibial bone was stored with surrounding tissue at $-70^{\circ} \mathrm{C}$ until the $\mathrm{Ca}$ and $\mathrm{P}$ analysis.

\section{Analytical Methods}

$\mathrm{Ca}$ and $\mathrm{P}$ Contents in Tibia: The left tibial bone was cleaned of surrounding tissue, and dried at $110^{\circ} \mathrm{C}$ for $12 \mathrm{hr}$. Dried samples were ashed at $680^{\circ} \mathrm{C}$ for $24 \mathrm{hr}$. Ashed samples were dissolved by $1.1 \mathrm{ml}$ of $6 \mathrm{~N} \mathrm{HCl}$, and diluted appropriately with distilled water for measurement. $\mathrm{Ca}$ and $\mathrm{P}$ contents in these samples were measured with commercial kits [Ca-C-test Wako; Wako Pure Chemical Industries (Osaka, Japan), Phospha-C-test Wako; Wako Pure Chemical Industries].

Bone mineral density in tibia by peripheral quantitative computed tomography ( $p Q C T)$ : The bone mineral density at the metaphysis of the right tibia was estimated by using pQCT (model XCT research SA+; Stratec Medizintechnik, Pforzheim, Germany). The metaphysis was defined as $3 \mathrm{~mm}$ below the articular surface. The voxel size was $0.12 \times 0.12 \times 0.77 \mathrm{~mm}$. The trabecular bone density was calculated as the area not over $395 \mathrm{mg} / \mathrm{cm}^{3}$.

Urinary DPD and Creatinine: Urinary DPD from the 24-hr sample, collected during on day 24, was measured with the commercial kit (Osoteolinks-DPD; DS Pharma Biomedical, Osaka, Japan). Urinary creatinine of the same sample was measured with 7150 Hitachi Clinical Analyzer (Hitachi High-technologies, Tokyo, Japan).

Urinary Isoflavones and Creatinine: Urinary isoflavones in the 24-hr sample collected on day 53 were analyzed by using HPLC, as previously reported. ${ }^{25)}$ All samples were analyzed at SRL, Tokyo, Japan.

Two hundred $\mu \mathrm{l}$ of appropriately diluted urine sample was added to acetate buffer ( $\mathrm{pH} 4.5)$ containing $\beta$-glucuronidase and sulfatase (Nippon BioTest Laboratories, Tokyo, Japan) and incubated overnight at $37^{\circ} \mathrm{C}$. Released aglycone was extracted with ethyl acetate. The organic layer was isolated and dried under nitrogen stream at $40^{\circ} \mathrm{C}$. The residue was dissolved in a solvent containing methanol, acetonitrile, dimethyl sulfoxide, and water, and used for HPLC analysis. The isoflavones daidzein, glycitein, genistein, and equol were separated with a column-switching HPLC system (LC10Avp series; Shimadzu, Kyoto, Japan), and detected by UV absorption. The pre-column was Wakosil-2 RS $4.6 \times 50 \mathrm{~mm}$ (Wako Pure Chemical 
Industries). The main column was CAPCELL PAK AQ $4.6 \times 250 \mathrm{~mm}$ (Shiseido, Tokyo, Japan). The separate condition was isocratic, using phosphate buffer containing acetonitrile and sodium perchlorate as the mobile phase. Creatinine of the same sample was measured with 7170 Hitachi Clinical Analyzer (Hitachi High-technologies).

Serum Osteocalcin: Serum osteocalcin from blood sample, collected on day 19, was measured with the commercial kit (Rat-MID Osteocalcin ELISA, IDS Nordic a/s, Herlev, Denmark).

Statistical Analysis — Data were expressed as means and standard error of mean (SEM). For urinary isoflavone data, significant differences between OVX two groups were determined by unpaired $t$-test (Statview-J 5.0, SAS institute, Cary, NC, U.S.A.). For the other data, significant differences among three groups were determined by oneway analysis of variance (ANOVA) and Fisher's PLSD test as a post-hoc test (Statview-J 5.0, SAS institute). Differences were considered significant at $p<0.05$.

\section{RESULTS}

\section{Body Weight, Food Intake, and Uterine Weight}

The OVX-NFS and OVX-FS groups had significantly higher mean food intake and body weight than the Sham-NFS group, but were not significantly different from each other for those values (Table 3). The OVX-NFS and OVX-FS group had significantly lower uterine weight than the ShamNFS group, but were not significantly different from each other.

\section{Bone Density}

The trabecular bone density at the metaphysis of the tibia is shown in Fig. 1. Compared to the ShamNFS group, the trabecular bone density was significantly reduced in the OVX-NFS group. This reduction tended to be prevented in the OVX-FS group; the OVX-FS group tended to have higher trabecular bone density than the OVX-NFS group.

\section{Bone $\mathrm{Ca}$ and $\mathrm{P}$ Contents in the Tibia}

$\mathrm{Ca}$ and $\mathrm{P}$ contents in the tibia are shown in Fig. 2. Compared to the Sham-NFS group, Ca content in the tibia was significantly lower in the OVXNFS group. This reduction was significantly prevented in the OVX-FS group; the OVX-FS group had significantly higher Ca content in the tibia than the OVX-NFS group. Likewise, $\mathrm{P}$ content in the tibia was significantly reduced by OVX and significantly prevented in the OVX-FS group. The OVXFS group had a significantly higher $\mathrm{P}$ content in the tibia than the OVX-NFS group.

\section{Urinary DPD and Serum Osteocalcin}

The day-24 urinary excretion of DPD, a bone resorption marker, is shown in Fig. 3 in terms of its ratio to endogenous creatinine excretion. Compared to the Sham-NFS group, urinary excretion of DPD was significantly increased in the OVX-NFS group. This increase was significantly prevented in the OVX-FS group; the OVX-FS group had a significantly lower DPD level than the OVX-NFS group.

The day-19 serum osteocalcin, a bone formation marker, is shown in Fig. 4. Compared to the Sham-NFS group, serum osteocalcin was signifi-

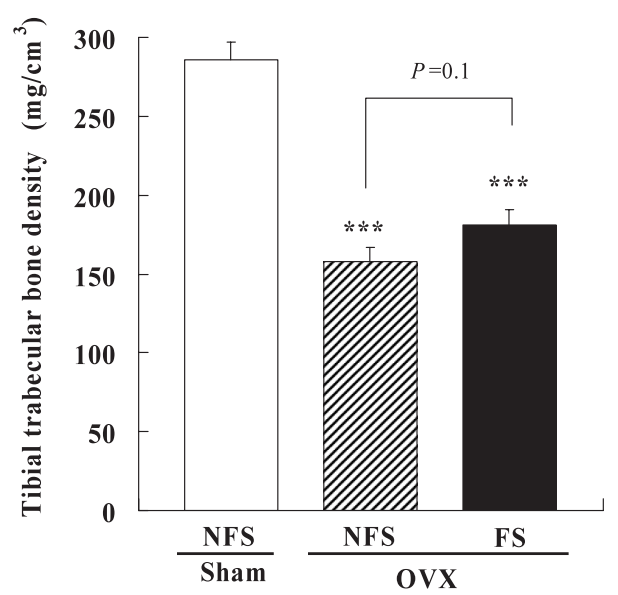

Fig. 1. Tibial Trabecular Bone Density in Rats Fed NFS or FS Diet for 57 Days

Values are means \pm SEM for ten rats. ${ }^{* * *} p<0.001$, Significantly different from Sham-NFS group.

Table 3. Final Body Weight, Food Intake, and Uterine Weight in Rats Fed NFS or FS Diet for 57 Days

\begin{tabular}{lccccc}
\hline \hline & \multicolumn{2}{c}{ Sham-NFS } & \multicolumn{2}{c}{ OVX-NFS } & \multicolumn{2}{c}{ OVX-FS } \\
\hline Final body weight $(\mathrm{g})$ & $261.4 \quad \pm 4.4 \mathrm{a}$ & 368.0 & $\pm 8.5 \mathrm{~b}$ & 366.5 & $\pm 7.9 \mathrm{~b}$ \\
Average food Intake $(\mathrm{g})$ & $13.6 \quad \pm 0.2 \mathrm{a}$ & $17.4 \quad \pm 0.5 \mathrm{~b}$ & 18.1 & $\pm 0.3 \mathrm{~b}$ \\
Uterine $(\mathrm{g} / 100 \mathrm{~g} \mathrm{BW})$ & $0.210 \pm 0.016 \mathrm{a}$ & $0.031 \pm 0.002 \mathrm{~b}$ & $0.033 \pm 0.003 \mathrm{~b}$ \\
\hline
\end{tabular}

Values are means \pm SEM for ten rats. Values in a row not sharing a common letter differ significantly $(p<0.001)$. 
(A)

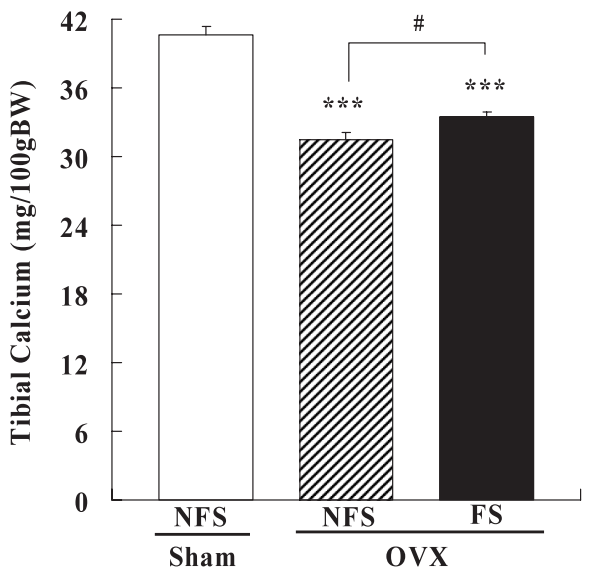

(B)

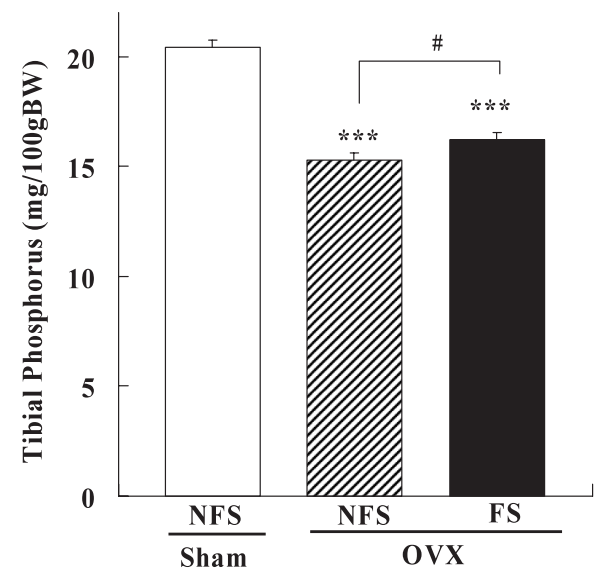

Fig. 2. Tibial Mineral Contents in Rats Fed NFS or FS Diet for 57 Days

A, Calcium; B, Phosphorus. Values are means \pm SEM for ten rats. ${ }^{* * *} p<0.001$, Significantly different from Sham-NFS group. $\# p<0.05$, Significantly different from OVX-NFS group.

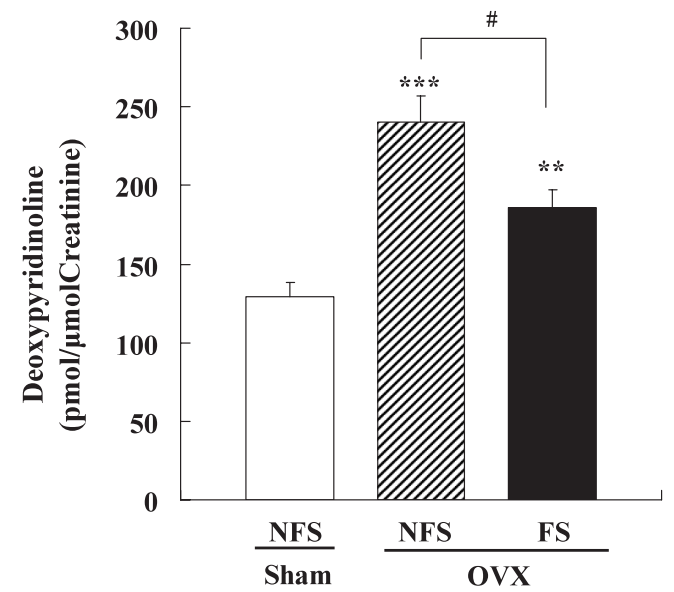

Fig. 3. Urinary deoxypyridinoline at day 24 in Rats Fed NFS or FS Diet

Values are means \pm SEM for ten rats. ${ }^{* * *} p<0.001,{ }^{* *} p<0.01$, Significantly different from Sham-NFS group. $\# p<0.05$, Significantly different from OVX-NFS group.

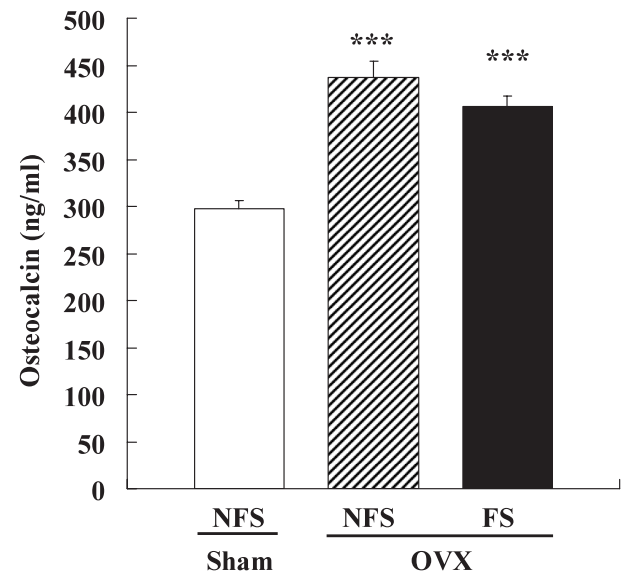

Fig. 4. Serum osteocalcin at day 19 in Rats Fed NFS or FS Diet

Values are means \pm SEM for ten rats. ${ }^{* * *} p<0.001$, Significantly different from Sham-NFS group.

cantly increased in the OVX-NFS group. There was no difference between the OVX-NFS and OVX-FS groups.

\section{Urinary Isoflavones}

The day-53 urinary excretion of isoflavones in the OVX groups is shown in Fig. 5 in terms of their ratios to endogenous creatinine excretion. The urinary daidzein level in OVX-FS group was significantly higher than that in OVX-NFS group. Genistein and total isoflavone level in OVX-FS group also tended to be higher than those in OVX-NFS group ( $p=0.1$ for both). The amount of total isoflavone was estimated as the sum of genistein, daidzein, glycitein, and equol. Glycitein and equol levels didn't differ significantly between the two groups.

\section{DISCUSSION}

The purpose of this study was to compare the effects of prolonged dietary intake of FS and NFS on bone metabolism, using OVX rats. Compared to the Sham-NFS group, the trabecular bone density and mineral contents in the tibia of rats in the OVXNFS group were significantly reduced. However, these reductions were prevented in the rats fed FS for 8 weeks. This result suggested that FS exhibited more effective isoflavone action than did NFS.

The bone loss caused by OVX has been reported to be prevented by isoflavone glucoside administration. ${ }^{26)}$ Arjmandji et al. $^{27)}$ reported the boneprotective effect of dietary ingestion of isoflavone 
(A)

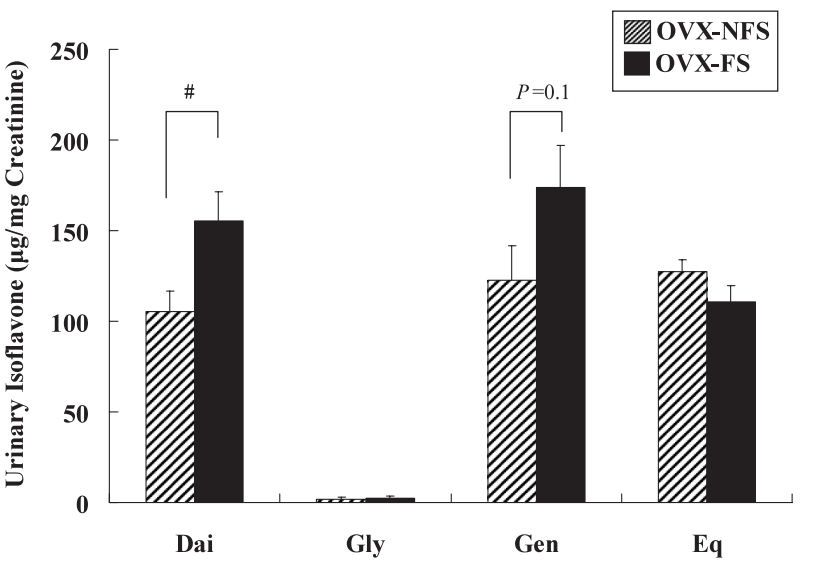

(B)

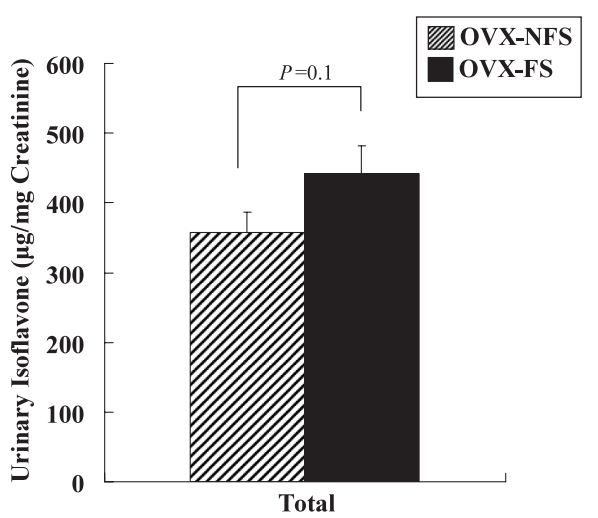

Fig. 5. Urinary isoflavones at day 53 in Rats Fed NFS or FS Diet

A, respective isoflavones; B, Total isoflavone. Values are means \pm SEM for ten rats. $\# p<0.05$, Significantly different from OVX-NFS group. Dai, Daidzein; Gly, Glycitein; Gen, Genistein; Eq, Equol. Total isoflavone: Dai, Gly, Gen, Eq.

glucoside-rich soy protein. The bone-protective effects of isoflavone aglycone have also been reported. Mathey et $a l .{ }^{28)}$ reported that isoflavone aglycone administration improved bone mineral density in OVX rats. Lien et al. ${ }^{29)}$ reported that dietary ingestion of aglycone had a favorable effect on bone mineral contents in OVX rats. In addition, Kawakami et al. ${ }^{30)}$ compared the effects of aglycone and glycoside on lipid metabolism in rats and suggested the superiority of aglycone. These previous reports agree with the results of the present study, in which prolonged dietary intake of FS showed protective effects on bone loss in OVX rats. The effects of FS appeared to be greater than those of NFS.

In this study, an 8-week intake of FS showed protective effects against bone loss. Mathey et al. ${ }^{31)}$ reported that a 12 -week intake of isoflavone at the same level as that in the present study $(228 \mu \mathrm{mol} / 100 \mathrm{~g}=0.06 \% \mathrm{w} / \mathrm{w}$ diet $)$ improved femoral bone strength. A longer intake of FS may result in even more improvement of bone strength.

In our preliminary study, a 4-week intake of FS tended to prevent the loss of mineral contents, but not at a significant level (data not shown). In general, the bone-sparing effects were reported after more than a 4-week intake. Deyhim et al. ${ }^{32}$ ) reported that at a low dose (about 0.014\%), isoflavones had no effect on bone mineral contents in such a short time. Using the same dose of isoflavones as we used in the present study, Hidaka et al. ${ }^{33)}$ reported that a 7 -week intake improved the tibial bone density. Therefore, we extended the intake period to 8 weeks in this study and clearly confirmed the bone-sparing effects of FS.
Food intake and body weight were significantly higher in the OVX group than in the Sham group. In general, OVX has been reported to cause excessive eating. ${ }^{34)}$ However, the bone density and mineral contents were significantly lower in the OVX group than in the Sham group. This established that the bone loss was definitely caused by OVX. Moreover, the primary goal of this study was to compare the effect on bone of FS and NFS, and the food intake of the OVX-FS and OVX-NFS groups was not significantly different.

OVX is commonly known to induce excessive bone resorption and formation in rats. ${ }^{35)}$ Bone resorption exceeds formation, thereby causing bone loss. That is, a drastic change in bone turnover markers is observed in advance of change in bone mineral contents, especially shortly after OVX. Picherit et al. ${ }^{36)}$ reported that the urine DPD level increased by 1.5 -fold at 5.7 weeks after OVX and returned to the baseline at 11 weeks. Serum osteocalcin level also increased by 1.6 -fold at 5.7 weeks, and returned to the baseline at 17 weeks. Therefore, in this study, the bone turnover markers were measured at 5 weeks after OVX ( $=3$ weeks after the start of dietary intake) to properly evaluate the changes. Urinary DPD results in this study agree with this previous knowledge. Compared to the Sham-NFS group, the urinary DPD level was significantly increased in the OVX-NFS group. This increase was significantly prevented in the rats fed FS, suggesting that dietary intake of FS inhibits the excessive bone resorption induced by OVX. We also measured serum osteocalcin, as a bone formation marker and found that it was significantly increased by OVX, but there was no difference between the OVX- 
FS and OVX-NFS groups. Therefore, we speculated that FS exerted bone-protective effects mostly through preventing the excessive bone resorption caused by OVX. On the other hand, isoflavones have been reported to affect bone resorption and formation in vitro. ${ }^{37-40)}$ Some authors have suggested that the actions of daidzein and genistein are slightly different, ${ }^{41)}$ and the detailed mechanism of their action is still unclear. Further studies on the mechanism of the bone protective effects by FS are necessary.

Urinary isoflavone levels showed a trend of being higher in rats fed FS for 8 weeks than in rats fed NFS. It was suggested that the bone-protective effects of FS may result from higher absorption of isoflavone aglycone. Kawakami et al. ${ }^{30)}$ also reported that the serum isoflavone level was higher in rats fed an aglycone-rich diet than in rats fed a glucoside-rich diet, speculating that this increase might be attributed to the beneficial effects on lipid metabolism. In this study, FS and NFS had equal amounts of fat, protein, $\mathrm{Ca}$, and $\mathrm{P}$ but different isoflavone types. Therefore, it was speculated FS enabled the higher absorption of isoflavone because its isoflavones were in the aglycone form. Of course, the two diets were also different in the decomposition level of their protein contents. Proteins in FS were mostly degraded, with largely free amino acids. On the other hand, proteins in NFS were kept as proteins with few free amino acids. To our knowledge, there have been few studies reporting that free amino acids affect the absorption of isoflavones. Further studies should be done, with careful consideration of what other factors might affect the absorption of isoflavones.

In conclusion, this study revealed that prolonged intake of FS prevented excessive bone resorption and subsequent bone loss in OVX rats. It was also suggested that these beneficial effects on bone resulted from the higher absorption of aglycone-form isoflavones.

Acknowledgements We would like to thank Mr. M Sakurai and Mr. H Matoba of Ajinomoto Co., Inc. for their cooperation in producing FS and NFS.

\section{REFERENCES}

1) Richelson, L. S., Wahner, H. W., Melton III, L. J. and Riggs, B. L. (1984) Relative contributions of aging and estrogen deficiency to postmenopausal bone loss. N. Engl. J. Med., 311, 1273-1275.
2) Rossouw, J. E., Anderson, G. L., Prentice, R. L., Lacroix, A. Z., Kooperberg, C., Stefanick, M. L., Jackson, R. D., Beresford, S. A. A., Howard, B. V., Johnson, K. C., Kotchen, J. M. and Ockene, J. (2002) Risks and Benefits of Estrogen Plus Progestin in Healthy Postmenopausal Women: Principal Results From the Women's Health Initiative Randomized Controlled Trial. JAMA, 288, 321-333.

3) Hulley, S., Furberg, C., Barrett-Connor, E., Cauley, J., Grady, D., Haskell, W., Knopp, R., Lowery, M., Satterfield, S., Schrott, H., Vittinghoff, E. and Hunninghake, D. (2002) Noncardiovascular Disease Outcomes During 6.8 Years of Hormone Therapy: Heart and Estrogen/Progestin Replacement Study Follow-up (HERS II). JAMA, 288, 58-64.

4) Kuiper, G. G. J. M., Lemmen, J. G., Carlsson, B., Corton, J. C., Safe, S. H., Van Der Saag, P. T., Van Der Burg, B. and Gustafsson, J.-A. (1998) Interaction of Estrogenic Chemicals and Phytoestrogens with Estrogen Receptor beta. Endocrinology, 139, 4252-4263.

5) Ishimi, Y., Miyaura, C., Ohmura, M., Onoe, Y., Sato, T., Uchiyama, Y., Ito, M., Wang, X., Suda, T. and Ikegami, S. (1999) Selective Effects of Genistein, a Soybean Isoflavone, on $\beta$-Lymphopoiesis and Bone Loss Caused by Estrogen Deficiency. Endocrinology, 140, 1893-1900.

6) Ishida, H., Uesugi, T., Hirai, K., Toda, T., Nukaya, H., Yokotsuka, K. and Tsuji, K. (1998) Preventive effects of the plant isoflavones, daidzin and genistin, on bone loss in ovariectomized rats fed a calciumdeficient diet. Biol. Pharm. Bull., 21, 62-66.

7) Morabito, N., Crisafulli, A., Vergara, C., Gaudio, A., Lasco, A., Frisina, N., D'anna, R., Corrado, F., Pizzoleo, M. A., Cincotta, M., Altavilla, D., Ientile, R. and Squadrito, F. (2002) Effects of Genistein and Hormone-Replacement Therapy on Bone Loss in Early Postmenopausal Women: A Randomized Double-Blind Placebo-Controlled Study. J. Bone Miner. Res., 17, 1904-1912.

8) Setchell, K. D. R. and Cassidy, A. (1999) Dietary Isoflavones: Biological Effects and Relevance to Human Health. J. Nutr., 129, 758-767.

9) Wang, H. and Murphy, P. A. (1994) Isoflavone Content in Commercial Soybean Foods. J. Agric. Food Chem., 42, 1666-1673.

10) Piskula, M. K., Yamakoshi, J. and Iwai, Y. (1999) Daidzein and genistein but not their glucosides are absorbed from the rat stomach. FEBS Lett., 447, 287-291.

11) Setchell, K. D., Brown, N. M., Zimmer-Nechemias, L., Brashear, W. T., Wolfe, B. E., Kirschner, A. S., and Heubi, J. E. (2002) Evidence for lack of absorp- 
tion of soy isoflavone glycosides in humans, supporting the crucial role of intestinal metabolism for bioavailability. Am. J. Clin. Nutr., 76, 447-453.

12) Cassidy, A. (2006) Factors Affecting the Bioavailability of Soy Isoflavones in Humans. J. AOAC Int., 89, 1182-1188.

13) Izumi, T., Piskula, M. K., Osawa, S., Obata, A., Tobe, K., Saito, M., Kataoka, S., Kubota, Y. and Kikuchi, M. (2000) Soy isoflavone aglycones are absorbed faster and in higher amounts than their glucosides in humans. J. Nutr., 130, 1695-1699.

14) Kano, M., Takayanagi, T., Harada, K., Sawada, S. and Ishikawa, F. (2006) Bioavailability of Isoflavones after Ingestion of Soy Beverages in Healthy Adults. J. Nutr., 136, 2291-2296.

15) Richelle, M., Pridmore-Merten, S., Bodenstab, S., Enslen, M. and Offord, E. A. (2002) Hydrolysis of Isoflavone Glycosides to Aglycones by betaGlycosidase Does Not Alter Plasma and Urine Isoflavone Pharmacokinetics in Postmenopausal Women. J. Nutr., 132, 2587-2592.

16) Zubik, L. and Meydani, M. (2003) Bioavailability of soybean isoflavones from aglycone and glucoside forms in American women. Am. J. Clin. Nutr., 77, 1459-1465.

17) Setchell, K. D., Brown, N. M., Desai, P. B., ZimmerNechimias, L., Wolfe, B., Jakate, A. S., Creutzinger, V. and Heubi, J. E. (2003) Bioavailability, disposition, and dose-response effects of soy isoflavones when consumed by healthy women at physiologically typical dietary intakes. J. Nutr., 133, 10271035.

18) Cassidy, A., Brown, J. E., Hawdon, A., Faughnan, M. S., King, L. J., Millward, J., Zimmer-Nechemias, L., Wolfe, B. and Setchell, K. D. R. (2006) Factors affecting the bioavailability of soy isoflavones in humans after ingestion of physiologically relevant levels from different soy foods. J. Nutr., 136, 45-51.

19) Holzapfel, W. H., Haberer, P., Snel, J., Schillinger, U. and Huis In't Veld, J. H. J. (1998) Overview of gut flora and probiotics. Int. J. Food Microbiol., 41, 85-101.

20) Sudo, N. (2005) Stress and Intestinal Flora. J. Intest. Microbiol., 19, 25-29 (in Japanese).

21) Wang, L.-J., Yin, L.-J., Li, D., Zou, L., Saito, M., Tatsumi, E. and Li, L.-T. (2007) Influences of processing and $\mathrm{NaCl}$ supplementation on isoflavone contents and composition during douchi manufacturing. Food Chem., 101, 1247-1253.

22) Toda, T., Tamura, J. and Okuhira, T. (1997) Isoflavone content in commercial soybean foods. Foods \& Food Ingred. J. Jpn., 172, 83-89 (in Japanese).
23) Murphy, P. A., Song, T., Buseman, G., Barua, K., Beecher, G. R., Trainer, D. and Holden, J. (1999) Isoflavones in Retail and Institutional Soy Foods. $J$. Agric. Food Chem., 47, 2697-2704.

24) Kudou, S., Fleury, Y. and Welti, D. (1991) Malonylisoflavone glycosides in soybean seeds (Glycine max MERILL). Agric. Biol. Chem., 55, 2227-2233.

25) Yamazaki, N., Gondou, K., Miyagawa, H., Kawagoe, N., Enoki, S. and Kubono, K. (2004) Determination of soybean isoflavones in human and urine. In 7th annual meeting of the Japanese Society for Complementary and Alternative Medicine, October 2004, Kanazawa, Japan P35, p. 89.

26) Uesugi, T., Toda, T., Tsuji, K. and Ishida, H. (2001) Comparative study on reduction of bone loss and lipid metabolism abnormality in ovariectomized rats by soy isoflavones, daidzin, genistin, and glycitin. Biol. Pharm. Bull., 24, 368-372.

27) Arjmandi, B. H., Birnbaum, R., Goyal, N. V., Getlinger, M. J., Juma, S., Alekel, L., Hasler, C. M., Drum, M. L., Hollis, B. W. and Kukreja, S. C. (1998) Bone-sparing effect of soy protein in ovarian hormone-deficient rats is related to its isoflavone content. Am. J. Clin. Nutr., 68, 1364S-1368S.

28) Mathey, J., Mardon, J., Fokialakis, N., Puel, C., Kati-Coulibaly, S., Mitakou, S., Bennetau-Pelissero, C., Lamothe, V., Davicco, M., Lebecque, P., Horcajada, M. and Coxam, V. (2007) Modulation of soy isoflavones bioavailability and subsequent effects on bone health in ovariectomized rats: the case for equol. Osteoporos Int., 18, 671-679.

29) Lien, T. F., Chen, W., Hsu, Y. L., Chen, H. L. and Chiou, R. Y. Y. (2006) Influence of Soy Aglycon Isoflavones on Bone-Related Traits and Lens Protein Characteristics of Ovariectomized Rats and Bioactivity Performance of Osteoprogenitor Cells. J. Agric. Food Chem., 54, 8027-8032.

30) Kawakami, Y., Tsurugasaki, W., Nakamura, S. and Osada, K. (2005) Comparison of regulative functions between dietary soy isoflavones aglycone and glucoside on lipid metabolism in rats fed cholesterol. J. Nutr. Biochem., 16, 205-212.

31) Mathey, J., Puel, C., Kati-Coulibaly, S., BennetauPelissero, C., Davicco, M. J., Lebecque, P., Horcajada, M. N. and Coxam, V. (2004) Fructooligosaccharides Maximize Bone-Sparing Effects of Soy Isoflavone-Enriched Diet in the Ovariectomized Rat. Calcif. Tissue Int., 75, 169-179.

32) Deyhim, F., Stoecker, B. J., Brusewitz, G. H. and Arjmandi, B. H. (2003) The effects of estrogen depletion and isoflavones on bone metabolism in rats. Nutr. Res., 23, 123-130.

33) Hidaka, S., Okamoto, Y., Miyazaki, K. and Uesugi, 
T. (2003) Evaluation of a soybean product fujiflavone P40 as an antiosteoporotic agent in rats. Phytother. Res., 17, 112-119.

34) Wronski, T., Schenk, P., Cintrón, M. and Walsh, C. (1987) Effect of body weight on osteopenia in ovariectomized rats. Calcif. Tissue Int., 40, 155159.

35) Wronski, T., Cintrón, M. and Dann, L. (1988) Temporal relationship between bone loss and increased bone turnover in ovariectomized rats. Calcif. Tissue Int., 43, 179-183.

36) Picherit, C., Bennetau-Pelissero, C., Chanteranne, B., Lebecque, P., Davicco, M.-J., Barlet, J.-P. and Coxam, V. (2001) Soybean Isoflavones DoseDependently Reduce Bone Turnover but Do Not Reverse Established Osteopenia in Adult Ovariectomized Rats. J. Nutr., 131, 723-728.

37) Okamoto, F., Okabe, K. and Kajiya, H. (2001) Genistein, a soybean isoflavone, inhibits inward rectifier K+ channels in rat osteoclasts. Jpn. J. Physiol.,
51, 501-509.

38) Sugimoto, E. and Yamaguchi, M. (2000) Stimulatory effect of daidzein in osteoblastic MC3T3-E1 cells. Biochem. Pharmacol., 59, 471-475.

39) Yamaguchi, M. and Sugimoto, E. (2000) Stimulatory effect of genistein and daidzein on protein synthesis in osteoblastic MC3T3-E1 cells: Activation of aminoacyl-tRNA synthetase. Mol. Cell. Biochem., 214, 97-102.

40) Gao, Y. H. and Yamaguchi, M. (2000) Suppressive effect of genistein on rat bone osteoclasts: involvement of protein kinase inhibition and protein tyrosine phosphatase activation. Int. J. Mol. Med., 5, 261-267.

41) Setchell, K. D. R. and Lydeking-Olsen, E. (2003) Dietary phytoestrogens and their effect on bone: evidence from in vitro and in vivo, human observational, and dietary intervention studies. Am. J. Clin. Nutr., 78, 593S-609S. 